\title{
Measuring sectoral diversification in an asymptotic multi-factor framework
}

\author{
Dirk Tasche*
}

July 2006

\begin{abstract}
We investigate a multi-factor extension of the asymptotic single risk factor (ASRF) model that underlies the capital charges of the "Basel II Accord". In this extended model, it is still possible to derive closed-form solutions for the risk contributions to Value-at-Risk and Expected Shortfall. As an application of the risk contribution formulae we introduce a new concept for a diversification measure. The use of this new measure is illustrated by an example calculated with a two-factor model. The results with this model indicate that, thanks to dependence on not fully correlated systematic sectors, there can be a substantial reduction of risk contributions by sectoral diversification effects.
\end{abstract}

\section{Introduction}

The Internal Ratings-Based Approach (IRBA) of Basel II (BCBS, 2006) is often regarded as a first step towards supervisory recognition of portfolio credit risk models for calculation of minimum capital requirements. IRBA capital formulae were derived within a special portfolio credit model, the so-called "Asymptotic Single Risk Factor Model" (ASRF model, Gordv, 2003). This model has the property that capital allocations to individual positions depend only upon the characteristics of these positions, but not upon the composition of the portfolio. This property of "portfolio invariance" allows for computational simplicity, in particular in that the required capital for a credit risk portfolio can be calculated in a bottom-up approach by determining capital requirements at the position level and adding them up. As a consequence, however, the

\footnotetext{
*Deutsche Bundesbank, Postfach 1006 02, 60006 Frankfurt am Main, Germany E-mail: dirk.tasche@gmx.net

The opinions expressed in this paper are those of the author and do not necessarily reflect views of Deutsche Bundesbank. The author thanks Michael Gordy, Katja Pluto, an anonymous referee and participants in the workshop "Concentration Risk in Credit Portfolios" (Eltville, November 2005) for useful comments on an earlier version of the paper.
} 
model can reflect neither name concentrations nor the sectoral structure of the portfolio (i.e., the distribution of borrowers across industry and geographic regions).

The model's inability to capture name concentrations entails a potential underestimation of the risk inherent in the portfolio, whereas its fault in recognizing the potential diversification effects following from the sectoral structure of the portfolio could result in an overestimation of portfolio risk. Overestimation of portfolio risk, however, can only occur if there is no significant change in the assigned asset correlations between the one-factor model and the multi-factor model that reflects the sectoral structure. In comparison with a multi-factor model where there are significantly higher average asset correlations, the ASRF model even can underestimate portfolio risk. As a consequence, whether the ASRF model underestimates the risk of a certain portfolio or not has to be examined on a case by case basis. The Basel Committee decided to deal in Pillar 2 of the Basel II Accord (BCBS, 2006) with the assessment of name risk concentration. As a consequence there is so far no automatism of extended regulatory capital requirements for risk concentrations, but banks will have to demonstrate to the supervisors that they have established appropriate procedures to keep concentrations under control. A quantitative way of tackling the name concentration issue was suggested in Emmer and Tasche (2005), for instance.

In the present paper, we suggest a minimal - in the spirit of Emmer and Tasche (2005) extension of the Basel II model that allows to study the effects of the sectoral structure on portfolio risk. Admitting several risk factors instead of a single factor only and applying the same transition to the limit as described in Gordv (2003), we arrive at versions of the model that remove the restriction of assuming a one-sector structure. Alternatively, our class of models can be regarded as special cases of the asymptotic models introduced by Lucas et al. (2001, Theorem $1)$.

As determining risk contributions or, economically speaking, capital requirements for assets, sectors or sub-portfolios, is a main purpose when using credit risk models, deriving exact formulae for risk contributions to "Value-at-risk" (VaR) and "Expected Shortfall" (ES) in the asymptotic multi-risk factors setting represents an important contribution of our paper to the subject. Our results complement results on the differentiation of VaR and ES presented in Gouriéroux et al. (2000), Lemus (1999), and Tasche (1999). In contrast to those papers, the results given below can be applied for calculating economic capital or contributions to economic capital without any further adaptation of the formulae. From a computational point of view, the resulting formulae are more demanding than in the one factor case, and - necessarily, as otherwise diversification effects could not be recognized - the resulting risk contributions are not portfolio invariant any longer.

As an application of the risk contribution formulae we introduce then a new concept for a measure of diversification, called diversification factor. This factor can be computed at portfolio as well as at sub-portfolio, sector or asset level, thus allowing the main concentrations to be identified. The use of these new factors is illustrated by an example calculated with a two-factor 
model. The results with this model indicate that there can be a substantial reduction of risk contributions by sectoral diversification effects.

The material presented here is closely related to work by Pvkhtin (2004) and Garcia Cespedes et al. (2006). Pykhtin describes an approximation of multi-factor models by single-factor models, thus transferring the computational simplicity of single-factor models to multi-factor models. Garcia Cespedes et al. propose "factor adjustments" to stand-alone capital charges in order to reflect diversification effects. As our results on the risk contribution formulae are not approximate but exact they could be used for benchmarking the results by Pvkhtin and Garcia Cespedes et al.

This paper is organized as follows: In Section 2 we introduce the class of models we are going to analyze and derive some basic properties. In Section 3 we briefly review the Euler allocation principle that justifies the use of partial derivatives as risk contributions and derive then the formulae for risk contributions to VaR and ES in the asymptotic multi-factor setting. A potential application of the risk contribution formulae for the purpose of identifying sources of risk concentration is suggested in Section 4 where a new concept for a diversification measure is introduced. Section 5 gives a numerical illustration of a potential application of the formulae and the diversification factor. We conclude with some summarizing comments in Section 6

\section{Asymptotic multi-factor models: basic properties}

The starting point for the factor models ${ }^{1}$ we are going to consider is a random variable $\tilde{L}(u)=$ $\tilde{L}\left(u_{1}, \ldots, u_{n}\right)$ that reflects the loss suffered from a portfolio of $n$ credit assets, with respective exposures $u_{i}$. The tilde indicates that we regard the original loss variable, without any approximation procedure. The variable $\tilde{L}(u)$ can be interpreted as the absolute loss, measured in units of some currency. Then the $u_{i}$ are absolute exposures ${ }^{2}$ and amounts of money. Alternatively, $\tilde{L}(u)$ can also be understood as relative loss, indicating the lost percentage of the sum of all exposures $^{3}$. In this case the $u_{i}$ are non-negative numbers without units that add up to 1 .

Formally, the original loss variable $\tilde{L}(u)$ is given as

$$
\tilde{L}(u)=\sum_{i=1}^{n} u_{i} \mathbf{1}_{D_{i}} .
$$

The term $\mathbf{1}_{D_{i}}$ is the default indicator variable for asset $i$, i.e. it takes the value of 1 if $i$ defaults and 0 if not. As a consequence, the sum in (2.1) will be built up with only those $u_{i}$ 's that relate

\footnotetext{
${ }^{1}$ See Bluhm et al. (2002) and the references therein for more information on credit risk models.

${ }^{2} u_{i}$ may also be thought as a face value multiplied with some factor that expresses the average loss rate in case of default.

${ }^{3}$ If the underlying absolute loss variable incorporates average loss rates in case of default, relative loss rather indicates the lost percentage of the sum of all exposures, weighted with their average loss rates.
} 
to defaulted assets $i$. For factor models, it is quite common to specify the default events $D_{i}$ by

$$
D_{i}=\left\{\sum_{j=1}^{k} \varrho_{i, j} S_{j}+\omega_{i} \xi_{i} \leq t_{i}\right\}, \quad i=1, \ldots, n,
$$

where the following holds for the involved constants and random variables:

- The random variables $S_{1}, \ldots, S_{k}$ are the systematic risk factors. They are assumed to capture the dependence of the default events. In general, we have $k \ll n$. Within this paper, we assume that the factor variables are standardized, i.e.

$$
\mathrm{E}\left[S_{j}\right]=0 \quad \text { and } \quad \operatorname{var}\left[S_{j}\right]=1, \quad j=1, \ldots, k .
$$

The $S_{1}, \ldots, S_{k}$ may be stochastically dependent, but they do not have do be.

- The random variables $\xi_{1}, \ldots, \xi_{n}$ are the idiosyncratic risk drivers. They are also standardized, i.e.

$$
\mathrm{E}\left[\xi_{i}\right]=0 \quad \text { and } \quad \operatorname{var}\left[\xi_{i}\right]=1, \quad i=1, \ldots, n .
$$

$\xi_{1}, \ldots, \xi_{n},\left(S_{1}, \ldots, S_{k}\right)$ are stochastically independent. As a consequence, conditional on $\left(S_{1}, \ldots, S_{k}\right)$, the default events $D_{i}, i=1, \ldots, n$ are independent.

- The constants $\varrho_{i, j}, i=1, \ldots, n, j=1, \ldots, k$ are the factor loadings of the systematic factors. We assume that

$$
\sum_{j=1, \ell=1}^{k} \varrho_{i, j} \varrho_{i, \ell} \operatorname{corr}\left[S_{j}, S_{\ell}\right] \leq 1, \quad i=1, \ldots, n .
$$

By (2.5) and the standardization assumption on the $S_{j}$ and $\xi_{i}$ the idiosyncratic loadings $\omega_{i}, i=\ldots, n$ are well defined by

$$
\omega_{i}=\sqrt{1-\sum_{j=1, \ell=1}^{k} \varrho_{i, j} \varrho_{i, \ell} \operatorname{corr}\left[S_{j}, S_{\ell}\right]} .
$$

As a further consequence of (2.5) and (2.6) and of the standardization assumptions also the asset values changes $\sum_{j=1}^{k} \varrho_{i, j} S_{j}+\omega_{i} \xi_{i}$ are standardized.

- The constant $t_{i}, i=1, \ldots, n$ is called default threshold. It can be thought as a critical loss in value of borrower $i$ 's assets that causes the borrower to default on asset $i$. It is common to derive $t_{i}$ from borrower $i$ 's (assumed to be known) probability of default $p_{i}$. Hence, we determine $t_{i}$ such that

$$
\mathrm{P}\left[D_{i}\right]=\mathrm{P}\left[\sum_{j=1}^{k} \varrho_{i, j} S_{j}+\omega_{i} \xi_{i} \leq t_{i}\right]=p_{i}, \quad i=1, \ldots n .
$$

When the idiosyncratic risk drivers $\xi_{i}$ and the factor variables are all standard normally distributed, also the asset value changes $\sum_{j=1}^{k} \varrho_{i, j} S_{j}+\omega_{i} \xi_{i}$ are standard normal. Let $\Phi$ denote the standard normal distribution function. By (2.7) we then have $p_{i}=\Phi\left(t_{i}\right)$. 
Assuming conditional independence of the default events, given the realizations of the systematic factors, entails by the law of large numbers that the loss variable $\tilde{L}(u)$ can be reasonably approximated by a modified loss variable $\hat{L}(u)$. The approximate loss variable $\hat{L}(u)$ then depends on the systematic factors only (cf. Gordv, 2003; Lucas et al., 2001). This is interpreted as elimination of the idiosyncratic risk by name diversification. In general, the quality of the approximation depends on conditions like the number of credit assets in the portfolio, the granularity of the portfolio, or the correlations of the asset value changes with the systematic factors. $\hat{L}(u)$ is obtained from $\tilde{L}(u)$ by replacing the default indicators $\mathbf{1}_{D_{i}}$ with their best predictors given the systematic factors, i.e. with the conditional probabilities $\mathrm{P}\left[D_{i} \mid\left(S_{1}, \ldots, S_{k}\right)\right]$. Hence $\hat{L}(u)$ is given by

$$
\hat{L}(u)=\sum_{i=1}^{n} u_{i} \mathrm{P}\left[D_{i} \mid\left(S_{1}, \ldots, S_{k}\right)\right] .
$$

Example 2.1 If the default events are given by (2.2) and the idiosyncratic risk drivers $\xi_{i}$ are standard normally distributed, then the approximate loss variable $\hat{L}(u)$ can be written as

$$
\hat{L}(u)=\sum_{i=1}^{n} u_{i} \Phi\left(\frac{t_{i}-\sum_{j=1}^{k} \varrho_{i, j} S_{j}}{\omega_{i}}\right) .
$$

Example 2.1 suggests to consider a - compared to (2.8) - slightly generalized loss variable L(u)

$$
L(u)=\sum_{i=1}^{n} u_{i} g_{i}(S)=\sum_{i=1}^{n} u_{i} g_{i}\left(S_{1}, \ldots, S_{k}\right),
$$

with $g_{i}: \mathbb{R}^{k} \rightarrow[0,1], i=1, \ldots, n$ decreasing ${ }^{4}$ at least in one (always the same) component of the vector argument. Considering the loss variable in this generalized form might be of interest, in particular, when losses are measured mark-to-market.

When investigating the generalized loss variable $L(u)$ we will need some technical conditions and notations as specified in the following assumption and (2.12a), (2.12b) and (2.12c).

\section{Assumption 2.2}

1. The exposures $u_{i}, i=1, \ldots, n$ in definition (2.10) are non-negative.

2. For any fixed $(k-1)$-tuple $\left(s_{2}, \ldots, s_{k}\right)$ the mapping

$$
s_{1} \mapsto \sum_{i=1}^{n} u_{i} g_{i}\left(s_{1}, \ldots, s_{k}\right), \quad \mathbb{R} \rightarrow[0, \infty[
$$

is strictly decreasing, continuous, and onto $] 0, \mathcal{U}\left[\right.$, with $\mathcal{U}$ defined by $\mathcal{U}=\sum_{i=1}^{n} u_{i}$.

\footnotetext{
${ }^{4}$ The results of this paper hold also when "decreasing" is replaced by "increasing". Some of the formulae then must be appropriately adapted.
} 
3. There is a conditional density $h\left(s_{1} \mid s_{2}, \ldots, s_{k}\right)$ of $S_{1}$ given $\left(S_{2}, \ldots, S_{k}\right)$.

The condition that the mapping from (2.11) is onto ]0, $\mathcal{U}$ [is, in particular, satisfied when $L(u)$ is specified as in Example 2.1 with $\varrho_{i, 1}>0$ for all $i=1, \ldots, n$. The condition, however, could be dispensed with, at the price of having more complexity in the following results on densities and risk contributions. For the sake of a more concise notation we define

$$
\widetilde{S}=\left(S_{2}, \ldots, S_{k}\right), \quad \widetilde{s}=\left(s_{2}, \ldots, s_{k}\right) .
$$

The distribution $\mathrm{P} \widetilde{S}^{-1}$ of $\widetilde{S}$ is then given by the relation

$$
\mathrm{P} \widetilde{S}^{-1}(A)=\mathrm{P}[\widetilde{S} \in A]
$$

that holds for all Borel-sets $A \subset \mathbb{R}^{k-1}$. In particular, if $\widetilde{S}$ has a density $\phi$, then integration with respect to the distribution of $\widetilde{S}$ can be expressed as $\mathrm{P} \widetilde{S}^{-1}(d \widetilde{s})=\phi(\widetilde{s}) d \widetilde{s}$. For fixed $u$ write

$$
G(v, \widetilde{s})=\sum_{j=1}^{n} u_{j} g_{j}(v, \widetilde{s})
$$

By Assumption 2.2] then, for fixed $\widetilde{s}, v \mapsto G(v, \widetilde{s})$ is invertible. Write $G(\cdot, \widetilde{s})^{-1}$ for the inverse function of $v \mapsto G(v, \widetilde{s})$. Write additionally $G(\cdot, \widetilde{s})^{-1}(0)=\infty$ and $G(\cdot, \widetilde{s})^{-1}(z)=-\infty$ for $z \geq \mathcal{U}$.

Having fixed the assumptions and notations, we can prove a result on the calculation of moments that in particular implies that the distribution of the generalized loss variable $L(u)$ has a density. Moreover, Proposition 2.3 will be applied, in the context of model (2.10), for identifying the VaR contributions as conditional expectations.

Proposition 2.3 Let $F:[0,1] \rightarrow \mathbb{R}$ be arbitrary and $L(u)$ be the loss variable defined by (2.10). Then, under Assumption ${ }^{5}$ [2.2, for any $0 \leq z \leq \mathcal{U}$ and $i \in\{1, \ldots, n\}$ we have:

$$
\mathrm{E}\left[F\left(g_{i}(S)\right) \mathbf{1}_{\{L(u) \leq z\}}\right]=-\int_{0}^{z} \mathrm{E}\left[\frac{F\left(g_{i}\left(G(\cdot, \widetilde{S})^{-1}(t), \widetilde{S}\right)\right) h\left(G(\cdot, \widetilde{S})^{-1}(t) \mid \widetilde{S}\right)}{\left.\frac{\partial}{\partial v} G(v, \widetilde{S})\right|_{v=G(\cdot, \widetilde{S})^{-1}(t)}}\right] d t
$$

\section{Proof.}

$$
\mathrm{E}\left[F\left(g_{i}(S)\right) \mathbf{1}_{\{L(u) \leq z\}}\right]=\int \mathrm{E}\left[F\left(g_{i}\left(S_{1}, \widetilde{S}\right)\right) \mathbf{1}_{\left\{G\left(S_{1}, \widetilde{S}\right) \leq z\right\}} \mid \widetilde{S}=\widetilde{s}\right] \mathrm{P}^{-1}(d \widetilde{s})
$$

(taking into account $G(\cdot, \widetilde{s})^{-1}(0)=\infty$ )

$$
=\iint_{G(\cdot, \widetilde{s})^{-1}(z)}^{\infty} F\left(g_{i}(y, \widetilde{s})\right) h(y \mid \widetilde{s}) d y \mathrm{P}^{-1}(d \widetilde{s})
$$

\footnotetext{
${ }^{5}$ In order to keep the representation of the results as intuitive and clear as possible here and in the following the proofs will not be rigorous but rather consist of calculations without consideration of continuity, differentiability etc. Moreover, for some of the results, additional assumptions on existence of moments etc. must be made.
} 
(substituting $\left.y=G(\cdot, \widetilde{s})^{-1}(t)\right)$

$$
=-\iint_{0}^{z} \frac{F\left(g_{i}\left(G(\cdot, \widetilde{s})^{-1}(t), \widetilde{s}\right)\right) h\left(G(\cdot, \widetilde{s})^{-1}(t) \mid \widetilde{s}\right)}{\left.\frac{\partial}{\partial v} G(v, \widetilde{S})\right|_{v=G(\cdot, \widetilde{S})^{-1}(t)}} d t \mathrm{P}^{-1}(d \widetilde{s}) .
$$

The assertion follows by applying Fubini's theorem.

The choice $F=1$ in Proposition 2.3 implies the existence of a density for the distribution of the generalized loss variable:

Corollary 2.4 Under Assumption 2.2, the loss variable $L(u)$ from (2.10) has the density $f_{L(u)}$ : ] $0, \mathcal{U}[\rightarrow[0, \infty[$, defined by

$$
\left.f_{L(u)}(t)=-\mathrm{E}\left[\frac{h\left(G(\cdot, \widetilde{S})^{-1}(t) \mid \widetilde{S}\right)}{\left.\frac{\partial}{\partial v} G(v, \widetilde{S})\right|_{v=G(\cdot, \widetilde{S})^{-1}(t)}}\right], \quad t \in\right] 0, \mathcal{U}[.
$$

Note that Gordv's (2003) ASRF (Asymptotic Single Risk Factor) model is a special case of 2.8) with $k=1$. Then the expectation in (2.14) disappears, and the density $h$ and the inverse of $G$ do not depend on $\widetilde{s}$. Nevertheless, in the case of non-constant asset correlations or probabilities of default the calculation of the density of $L(u)$ will involve numerical inversion of $G$ even in the simple ASRF case.

In case that a closed-form representation of the conditional distribution of $S_{1}$ given $\widetilde{S}$ is known (e.g. if $S$ is jointly normally distributed), Equation (2.13a) (with $F=1$ ) immediately yields a more efficient way to calculate the distribution function of $L(u)$ than Corollary 2.4 does. The reason is that application of Corollary 2.4 would require evaluation of a $k$-dimensional integral if $S$ had a density, whereas the application of the following Proposition 2.5 would only require evaluation of a $(k-1)$-dimensional integral.

Proposition 2.5 Under Assumption 2.2. the distribution function of the loss variable $L(u)$ as given in (2.10) can be calculated by means of

$$
\mathrm{P}[L(u) \leq z]=\int \mathrm{P}\left[S_{1} \geq G(\cdot, \widetilde{S})^{-1}(z) \mid \widetilde{S}=\widetilde{s}\right] \mathrm{P} \widetilde{S}^{-1}(d \widetilde{s})
$$

Define, for $\alpha \in(0,1)$ and any real random variable $X$, the $\alpha$-quantile of $X$ by

$$
q_{\alpha}(X)=\min \{x: \mathrm{P}[X \leq x] \geq \alpha\}
$$

Quantiles at high levels (e.g. 99.9\%) are popular metrics for determining the economic capital of portfolios. Within the financial community, the $\alpha$-quantile of a loss distribution is commonly called Value-at-Risk (VaR) at level $\alpha$. In case of the generalized loss variable $L(u)$ the quantiles $q_{\alpha}(L(u))$ can be computed by numerical inversion of (2.15). 
We conclude this section by providing two alternative formulae for the calculation of another popular risk measure, the Expected Shortfall ${ }^{6}$, in the case of the asymptotic multi-factor model under consideration.

Remark 2.6 The Expected Shortfall $\mathrm{ES}_{\alpha}(L(u))=\mathrm{E}\left[L(u) \mid L(u) \geq q_{\alpha}(L(u))\right]$ at level $\alpha$ of the loss variable $L(u)$ from (2.10) can alternatively be calculated with recourse to Corollary 2.4 or to Proposition [2.5. Note that the existence of a density of the distribution of L(u) (Corollary 2.4) implies $\mathrm{P}\left[L(u) \geq q_{\alpha}(L(u))\right]=1-\alpha$. From Corollary 2.4 we can therefore derive

$$
\mathrm{E}\left[L(u) \mid L(u) \geq q_{\alpha}(L(u))\right]=-(1-\alpha)^{-1} \int_{q_{\alpha}(L(u))}^{\mathcal{U}} t \mathrm{E}\left[\frac{h\left(G(\cdot, \widetilde{S})^{-1}(t) \mid \widetilde{S}\right)}{\left.\frac{\partial}{\partial v} G(v, \widetilde{S})\right|_{v=G(\cdot, \widetilde{S})^{-1}(t)}}\right] d t .
$$

From Proposition [2.5 we obtain (by making use of the formula $\mathrm{E}[X]=\int_{0}^{\infty} \mathrm{P}[X \geq x] d x$ for $X \geq 0)$

$$
\begin{aligned}
& \mathrm{E}\left[L(u) \mid L(u) \geq q_{\alpha}(L(u))\right] \\
& =q_{\alpha}(L(u))+(1-\alpha)^{-1} \int_{q_{\alpha}(L(u))}^{\mathcal{U}} \int \mathrm{P}\left[S_{1} \leq G(\cdot, \widetilde{S})^{-1}(z) \mid \widetilde{S}=\widetilde{s}\right] \mathrm{P}^{-1}(d \widetilde{s}) d z .
\end{aligned}
$$

\section{Computing the risk contributions}

When economic capital for a portfolio is determined by means of a homogeneous risk measure, according to the Euler allocation principle - to be introduced in Section 3.1 - the risk contributions of assets should be calculated as partial derivatives of the portfolio-wide economic capital with respect to the exposures. In Section 3.2 we will then derive formulae for the derivatives of Value-at-Risk ${ }^{7}$ as defined by (2.16) and Expected Shortfall as defined in Remark [2.6] in the context of the asymptotic multi-factor model of Section 2

\subsection{Euler allocation}

Suppose that real-valued random variables $X_{1}, \ldots, X_{n}$ are given that stand for the profits and losses with the assets in a portfolio. Let $Y$ denote the portfolio-wide profit and loss, i.e. let

$$
Y=\sum_{i=1}^{n} X_{i}
$$

\footnotetext{
${ }^{6}$ See Acerbi and Tasche (2002) and the references given therein for more details on Expected Shortfall vs. Value-at-Risk. In particular, in case of discontinuous loss distributions the definition of ES has to be slightly modified in order to make it a risk measure superior to VaR.

${ }^{7}$ See Mausser and Rosen (2004) and the references therein for the practical issues when estimating VaR contributions from statistical samples.
} 
The economic capital EC required by the portfolio is determined with a risk measure $\rho$, i.e.

$$
\mathrm{EC}=\rho(Y) .
$$

Definition 3.1 If $\rho$ is a risk measure and $V, W$ are random variables such that the derivative $\left.\frac{d}{d h} \rho(h V+W)\right|_{h=0}$ exists, then

$$
\rho(V \mid W)=\left.\frac{d}{d h} \rho(h V+W)\right|_{h=0}
$$

is called contribution of $V$ to the risk of $W$ in respect of $\rho$.

It is natural to require that, in a portfolio with loss variable as in (3.1), the risk contributions add up to the portfolio EC. As we will see below, this property is closely related to a homogeneity property of the risk measure $\rho$.

Assumption 3.2 The risk measure $\rho$ is positively homogeneous, i.e.

$$
\rho(h Z)=h \rho(Z)
$$

for any random variable $Z$ in the definition set of $\rho$ and $h>0$.

This assumption seems very natural as long as the asset or portfolio under consideration is not significant compared to the market as a whole and is not subject to market liquidity risk.

If for every $i$ the contribution of $X_{i}$ to the risk of $Y$ exists, then we have by Euler's theorem on the representation of positively homogenous functions

$$
\rho(Y)=\sum_{i=1}^{n} \rho\left(X_{i} \mid Y\right) .
$$

Hence, as required, positive homogeneity implies that, within a portfolio, the risk contributions add up to the total risk. This additivity property is of high practical importance.

The decomposition of the portfolio risk $\rho$ as given by (3.3) is called Euler allocation. The use of the Euler allocation principle was justified by several authors with different reasonings:

- Patrik et al. (1999) argued from a practitioner's view emphasizing mainly the fact that the risk contributions according to the Euler principle by (3.3) naturally add up to the portfolio-wide economic capital.

- Litterman (1996) and Tasche (1999) pointed out that the Euler principle is fully compatible with economically sensible portfolio diagnostics and optimization.

- Denault (2001) derived the Euler principle by game-theoretic considerations. 
- In the context of capital allocation for insurance companies, Mvers and Read (2001) argued that applying the Euler principle to the expected "default value" (essentially $\mathrm{E}[\max (Y, 0)]$ ) of the insurance portfolio is most appropriate for deriving line-by-line surplus requirements.

- More recently Kalkbrener (2005) presented an axiomatic approach to capital allocation and risk contributions. One of his axioms requires that risk contributions do not exceed the corresponding stand-alone risks. From this axiom in connection with more technical conditions, in the context of sub-additive and positively homogeneous risk measures, Kalkbrener concluded that the Euler principle is the only allocation principle to be compatible with the "diversification"-axiom (see also Kalkbrener et al., 2004; Tasche, 2002).

\subsection{Partial derivatives of VaR and ES}

Before coming to the main result on the partial derivatives of VaR with respect to the exposures of the assets in the portfolio, we will shortly discuss the case of Gordv's (2003) ASRF model.

Example 3.3 In Gordd's (2003, cf. Proposition 4) ASRF model Equation (2.10) reads

$$
L(u)=\sum_{j=1}^{n} u_{j} g_{j}(S)
$$

where $S$, the single systematic factor, stands for a random variable that satisfies some additional conditions. The $g_{j}$ are strictly increasing and continuous functions. As a consequence, the terms $g_{j}(S)$ in (3.4) are comonotonic (see Section 4 for a definition) random variables. From the comonotonic additivity of $\operatorname{VaR}$ and $E S$ (see, e.g., Tasche, 200\%) follows then for any $\alpha \in(0,1)$ that

$$
q_{\alpha}(L(u))=\sum_{j=1}^{n} u_{j} q_{\alpha}\left(g_{j}(S)\right)
$$

and

$$
\mathrm{E}\left[L(u) \mid L(u) \geq q_{\alpha}(L(u))\right]=\sum_{j=1}^{n} u_{j} \mathrm{E}\left[g_{j}(S) \mid g_{j}(X) \geq q_{\alpha}\left(g_{j}(S)\right)\right] .
$$

As the right-hand sides of (3.5a) and (3.5b) are linear in the exposure vector $u$, applying the Euler allocation principle with partial derivatives with respect to the components of $u$ yields that the risk contributions to VaR or ES in the ASRF model model equal the corresponding stand-alone risks.

In the following, we compute the derivatives of VaR and ES in the context of an asymptotic multi-factor model as given by (2.10). The validity of the results is subject to technical conditions 
similar to those of Tasche (1999, Section 5). For reasons of readability of the text we do not discuss these conditions here in detail.

Write (slightly modifying the notation from (2.12c) but keeping (2.12a )

$$
G(v, \widetilde{s}, u)=\sum_{j=1}^{n} u_{j} g_{j}(v, \widetilde{s})
$$

as well as

$$
G_{(\widetilde{s}, u)}^{-1}(z)=G(\cdot, \widetilde{s}, u)^{-1}(z)
$$

Hence $G_{(\widetilde{s}, u)}^{-1}(z)$ denotes the solution $v^{*}$ of the equation

$$
G\left(v^{*}, \widetilde{s}, u\right)=z
$$

with fixed $\widetilde{s}$ and $u$. Note that existence and uniqueness of $G_{(\widetilde{s}, u)}^{-1}(z)$ is guaranteed by Assumption 2.2. Thus prepared, we can state the main result of this paper (Theorem 3.4), namely that in the asymptotic multi-factor model the risk contributions to VaR, calculated as partial derivatives, coincide with certain expectations conditional on the portfolio loss equalling VaR.

Theorem 3.4 Under Assumption [2.2 $(0,1)$ of the generalized loss variable $L(u)$ as defined in (2.10) are partially differentiable with respect to the portfolio weights $u_{i}$ of the single loss variables. The partial derivatives $\frac{\partial q_{\alpha}(u)}{\partial u_{i}}$ are given by

$$
\frac{\partial q_{\alpha}(u)}{\partial u_{i}}=\mathrm{E}\left[\frac{h\left(G_{(\widetilde{S}, u)}^{-1}\left(q_{\alpha}(u)\right) \mid \widetilde{S}\right)}{\left.\frac{\partial}{\partial v} G(v, \widetilde{S}, u)\right|_{v=G_{(\widetilde{S}, u)}^{-1}\left(q_{\alpha}(u)\right)}}\right]^{-1} \mathrm{E}\left[\frac{g_{i}\left(G_{(\widetilde{S}, u)}^{-1}\left(q_{\alpha}(u)\right), \widetilde{S}, u\right) h\left(G_{(\widetilde{S}, u)}^{-1}\left(q_{\alpha}(u)\right) \mid \widetilde{S}\right)}{\left.\frac{\partial}{\partial v} G(v, \widetilde{S}, u)\right|_{v=G_{(\widetilde{S}, u)}^{-1}\left(q_{\alpha}(u)\right)}}\right] .
$$

Proof. Fix $z \in] 0, \mathcal{U}[$ and observe from (3.6c) that

$$
G\left(G_{(\widetilde{s}, u)}^{-1}(z), \widetilde{s}, u\right)=z \text { for all } \widetilde{s}, u
$$

implies by $3.6 \mathrm{a}$

$$
\begin{aligned}
0 & \left.=\frac{\partial}{\partial u_{i}} G\left(G_{(\widetilde{s}, u)}^{-1}(z), \widetilde{s}, u\right)\right) \\
& =\left.\frac{\partial}{\partial u_{i}} G_{(\widetilde{s}, u)}^{-1}(z) \frac{\partial}{\partial v} G(v, \widetilde{s}, u)\right|_{v=G_{(\widetilde{s}, u)}^{-1}(z)}+\left.\frac{\partial}{\partial w_{i}} G\left(G_{(\widetilde{s}, u)}^{-1}(z), \widetilde{s}, w\right)\right|_{w=u} \\
& =\left.\frac{\partial}{\partial u_{i}} G_{(\widetilde{s}, u)}^{-1}(z) \frac{\partial}{\partial v} G(v, \widetilde{s}, u)\right|_{v=G_{(\widetilde{s}, u)}^{-1}(z)}+g_{i}\left(G_{(\widetilde{s}, u)}^{-1}(z), \widetilde{s}\right)
\end{aligned}
$$

\footnotetext{
${ }^{8}$ Some further technical conditions on uniform integrability of the random variables under consideration have to be required, cf. Tasche (1999, Lemma 5.3) for a similar result.
} 
and as a further consequence

$$
\frac{\partial}{\partial u_{i}} G_{(\widetilde{s}, u)}^{-1}(z)=-\frac{g_{i}\left(G_{(\widetilde{s}, u)}^{-1}(z), \widetilde{s}\right)}{\left.\frac{\partial}{\partial v} G(v, \widetilde{s}, u)\right|_{v=G_{(\widetilde{s}, u)}^{-1}(z)}}
$$

Additionally, we have

$$
\frac{\partial}{\partial z} G_{(\widetilde{s}, u)}^{-1}(z)=\left(\left.\frac{\partial}{\partial v} G(v, \widetilde{s}, u)\right|_{v=G_{(\widetilde{s}, u)}^{-1}(z)}\right)^{-1}
$$

Assuming existence ${ }^{9}$ of $\frac{\partial q_{\alpha}(u)}{\partial u_{i}}$, it can implicitly be determined as follows:

$$
\begin{aligned}
\alpha & =\mathrm{P}\left[L(u) \leq q_{\alpha}(u)\right] \\
& =\mathrm{E}\left[\mathrm{P}\left[S_{1} \geq G_{(\widetilde{S}, u)}^{-1}\left(q_{\alpha}(u)\right) \mid \widetilde{S}\right]\right] \\
& =\mathrm{E}\left[\int_{G_{(\widetilde{S}, u)}^{-1}\left(q_{\alpha}(u)\right)}^{\infty} h(y \mid \widetilde{S}) d y\right]
\end{aligned}
$$

implies

$$
0=-\mathrm{E}\left[\frac{\partial}{\partial u_{i}} G_{(\widetilde{S}, u)}^{-1}\left(q_{\alpha}(u)\right) h\left(G_{(\widetilde{S}, u)}^{-1}\left(q_{\alpha}(u)\right) \mid \widetilde{S}\right)\right]
$$

By (3.8a) and (3.8b) we obtain

$$
\begin{aligned}
\frac{\partial}{\partial u_{i}} G_{(\widetilde{S}, u)}^{-1}\left(q_{\alpha}(u)\right) & =\left.\frac{\partial}{\partial u_{i}} G_{(\widetilde{S}, u)}^{-1}(z)\right|_{z=q_{\alpha}(u)}+\left.\frac{\partial}{\partial z} G_{(\widetilde{S}, u)}^{-1}(z)\right|_{z=q_{\alpha}(u)} \frac{\partial q_{\alpha}(u)}{\partial u_{i}} \\
& =\left(\left.\frac{\partial}{\partial v} G(v, \widetilde{S}, u)\right|_{v=G_{(\widetilde{S}, u)}^{-1}\left(q_{\alpha}(u)\right)}\right)^{-1}\left(\frac{\partial q_{\alpha}(u)}{\partial u_{i}}-g_{i}\left(G_{(\widetilde{S}, u)}^{-1}\left(q_{\alpha}(u)\right), \widetilde{S}\right)\right) .
\end{aligned}
$$

Replacing $\frac{\partial}{\partial u_{i}} G_{(\widetilde{S}, u)}^{-1}\left(q_{\alpha}(u)\right)$ in (3.9) by the right-hand side of (3.10) and solving for $\frac{\partial q_{\alpha}(u)}{\partial u_{i}}$ yields the assertion.

Remark 3.5 Equation (3.7) may equivalently be written as

$$
\frac{\partial q_{\alpha}(L(u))}{\partial u_{i}}=\mathrm{E}\left[g_{i}(S) \mid L(u)=q_{\alpha}(L(u))\right] .
$$

This follows from Proposition 2.3 and Corollary 2.4. For by Corollary 2.4, we have for any $z \in] 0, \mathcal{U}[$

$$
\mathrm{E}\left[g_{i}(S) \mathbf{1}_{\{L(u) \leq z\}}\right]=-\int_{0}^{z} \mathrm{E}\left[g_{i}(S) \mid L(u)=t\right] \mathrm{E}\left[\frac{h\left(G(\cdot, \widetilde{S})^{-1}(t) \mid \widetilde{S}\right)}{\left.\frac{\partial}{\partial v} G(v, \widetilde{S})\right|_{v=G(\cdot, \widetilde{S})^{-1}(t)}}\right] d t .
$$

\footnotetext{
${ }^{9}$ Under appropriate smoothness and moment conditions, existence can be proven by means of the implicit function theorem.
} 
On the other hand, Proposition 2.3 implies with $F(x)=x$ that

$$
\mathrm{E}\left[g_{i}(S) \mathbf{1}_{\{L(u) \leq z\}}\right]=-\int_{0}^{z} \mathrm{E}\left[\frac{g_{i}\left(G(\cdot, \widetilde{S})^{-1}(t), \widetilde{S}\right) h\left(G(\cdot, \widetilde{S})^{-1}(t) \mid \widetilde{S}\right)}{\left.\frac{\partial}{\partial v} G(v, \widetilde{S})\right|_{v=G(\cdot, \widetilde{S})^{-1}(t)}}\right] d t
$$

Equating the right-hand sides of (3.12a) and (3.12b) respectively implies by taking the derivative with respect to $z$ and then letting $z=q_{\alpha}(L(u))$ that $\mathrm{E}\left[g_{i}(S) \mid L(u)=q_{\alpha}(L(u))\right]$ equals the righthand side of (3.7).

A result analogous to Theorem 3.4 for VaR holds for ES as the following corollary shows.

Corollary 3.6 Under Assumption 2.2. the Expected Shortfall risk measure $\mathrm{E}[L(u) \mid L(u) \geq$ $q_{\alpha}(u)$ ] (with $q_{\alpha}(u)=q_{\alpha}(L(u))$ ) of the generalized loss variable as defined in (2.10) is partially differentiable with respect to the weights $u_{i}$. The partial derivatives can be computed as

$$
\frac{\partial}{\partial u_{i}} \mathrm{E}\left[L(u) \mid L(u) \geq q_{\alpha}(u)\right]=\mathrm{E}\left[g_{i}(S) \mid L(u) \geq q_{\alpha}(u)\right], \quad i=1, \ldots, n .
$$

Proof. A straight-forward calculation as in the proof of Proposition 2.3 (see (2.13b) yields

$$
\begin{aligned}
& (1-\alpha) \frac{\partial}{\partial u_{i}} \mathrm{E}\left[L(u) \mid L(u) \geq q_{\alpha}(u)\right] \\
& =\frac{\partial}{\partial u_{i}}\left(\sum_{j=1}^{n} u_{j} \mathrm{E}\left[\int_{-\infty}^{G_{(\widetilde{S}, u)}^{-1}\left(q_{\alpha}(u)\right)} g_{j}(y, \widetilde{S}) h(y \mid \widetilde{S}) d y\right]\right) \\
& =\sum_{j=1}^{n} u_{j} \mathrm{E}\left[\frac{\partial}{\partial u_{i}} G_{(\widetilde{S}, u)}^{-1}\left(q_{\alpha}(u)\right) g_{j}\left(G_{(\widetilde{S}, u)}^{-1}\left(q_{\alpha}(u)\right), \widetilde{S}\right) h\left(G_{(\widetilde{S}, u)}^{-1}\left(q_{\alpha}(u)\right) \mid \widetilde{S}\right)\right] \\
& \quad+\mathrm{E}\left[g_{i}(S) \mathbf{1}_{\left\{L(u) \geq q_{\alpha}(u)\right\}}\right] .
\end{aligned}
$$


Making use of identity (3.10) and of the definition of $G_{(\widetilde{S}, u)}^{-1}$ (see (3.6a) and (3.6b) ) we obtain

$$
\begin{aligned}
& \sum_{j=1}^{n} u_{j} \mathrm{E}\left[\frac{\partial}{\partial u_{i}} G_{(\widetilde{S}, u)}^{-1}\left(q_{\alpha}(u)\right) g_{j}\left(G_{(\widetilde{S}, u)}^{-1}\left(q_{\alpha}(u)\right), \widetilde{S}\right) h\left(G_{(\widetilde{S}, u)}^{-1}\left(q_{\alpha}(u)\right) \mid \widetilde{S}\right)\right] \\
& =\frac{\partial q_{\alpha}(u)}{\partial u_{i}} \mathrm{E}\left[\frac{\left.\left(\sum_{j=1}^{n} u_{j} g_{j}\left(G_{(\widetilde{S}, u)}^{-1}\left(q_{\alpha}(u)\right), \widetilde{S}\right)\right) h\left(G_{(\widetilde{S}, u)}^{-1}\left(q_{\alpha}(u)\right) \mid \widetilde{S}\right)\right]}{\left.\frac{\partial}{\partial v} G(v, \widetilde{S}, u)\right|_{v=G_{(\widetilde{S}, u)}^{-1}\left(q_{\alpha}(u)\right)}}\right] \\
& \quad-\mathrm{E}\left[\frac{\left(\sum_{j=1}^{n} u_{j} g_{j}\left(G_{(\widetilde{S}, u)}^{-1}\left(q_{\alpha}(u)\right), \widetilde{S}\right)\right) g_{i}\left(G_{(\widetilde{S}, u)}^{-1}\left(q_{\alpha}(u)\right), \widetilde{S}\right) h\left(G_{(\widetilde{S}, u)}^{-1}\left(q_{\alpha}(u)\right) \mid \widetilde{S}\right)}{\left.\frac{\partial}{\partial v} G(v, \widetilde{S}, u)\right|_{v=G_{(\widetilde{S}, u)}^{-1}\left(q_{\alpha}(u)\right)}}\right] \\
& =\quad q_{\alpha}(u)\left\{\frac{\partial q_{\alpha}(u)}{\partial u_{i}} \mathrm{E}\left[\frac{h\left(G_{(\widetilde{S}, u)}^{-1}\left(q_{\alpha}(u)\right) \mid \widetilde{S}\right)}{\left.\left.\frac{\partial}{\partial v} G(v, \widetilde{S}, u)\right|_{v=G_{(\widetilde{S}, u)}^{-1}\left(q_{\alpha}(u)\right)}\right]}\right]\right\} \\
& \left.\quad-\mathrm{E}\left[\frac{g_{i}\left(G_{(\widetilde{S}, u)}^{-1}\left(q_{\alpha}(u)\right), \widetilde{S}\right) h\left(G_{(\widetilde{S}, u)}^{-1}\left(q_{\alpha}(u)\right) \mid \widetilde{S}\right)}{\left.\frac{\partial}{\partial v} G(v, \widetilde{S}, u)\right|_{v=G_{(\widetilde{S}, u)}^{-1}\left(q_{\alpha}(u)\right)}}\right]\right\} \\
& =0,
\end{aligned}
$$

by Theorem 3.4. By means of (3.14) this implies the assertion.

Remark 3.7 At first sight, formulae (3.11) and (3.13) look very much like corresponding formulae for the derivatives of VaR and ES in Gouriéroux et al. (2000), Lemus (1999), and Tasche (1999). Note, however, that those formulae were not derived in an asymptotic multi-factor setting like the ones here. On the other hand, the validity of the results by Gouriéroux et al. (2000), Lemus (1999), and Tasche (1999) is not restricted to the case of bounded loss variables of the assets. Therefore, the results from this paper and the earlier results complement each other.

\section{Defining a diversification measure}

During the last few years, with regard to applications, three properties of risk measures $\rho$ turned out to be potentially most important:

- Positive homogeneity. See Assumption 3.2 for the formal definition and some comments.

- Sub-additivity. Artzner et al. (1999, Axiom S) described sub-additivity with "a merger does not create extra risk". VaR as a risk measure is mainly criticized for lacking this property. Kalkbrener et al. (2004) pointed out that, in the context of bank-internal credit risk management, the following characterization of sub-additivity is more apposite. Subadditivity of a positively homogeneous risk measures is equivalent to the property that risk 
contributions are not larger than the corresponding stand-alone risks. Speaking in terms of Sub-section 3.1

$$
\rho(V+W) \leq \rho(V)+\rho(W) \text { for all } V, W \Leftrightarrow \rho(V \mid W) \leq \rho(V) \text { for all } V, W,
$$

if $\rho$ is positively homogeneous (Tasche, 2002, Proposition 2.5).

- Comonotonic additivity. In actuarial science, the concept of comonotonicity is wellknown as it supports easy and reasonably conservative representations of dependence structures (see, e.g., Dhaene et al., 2006). Random variables $V$ and $W$ are called comonotonic if they can be represented as non-decreasing functions of a third random variable $Z$, i.e.

$$
V=h_{V}(Z) \quad \text { and } \quad W=h_{W}(Z)
$$

for some non-decreasing functions $h_{V}, h_{W}$. As comonotonicity is implied if $V$ and $W$ are correlated with correlation coefficient 1, it generalizes the concept of linear dependence. A risk measure $\rho$ is called comonotonic additive if for any comonotonic random variables $V$ and $W$

$$
\rho(V+W)=\rho(V)+\rho(W) .
$$

Thus comonotonic additivity can be interpreted as a specification of the worst case scenarios for the sub-additivity (4.1): nothing worse can occur than comonotonic random variables - which seems quite natural.

Note that VaR is positively homogeneous and comonotonic additive but not sub-additive and that ES is positively homogeneous, comonotonic additive and sub-additive (see, e.g. Tasche, 2002). As a consequence, finding worst case scenarios for given marginal distributions of $V, W$ in (4.1) is easy in case of ES (take the comonotonic scenario) and non-trivial in case of VaR (see Embrechts et al., 2003; Luciano and Marena, 2003).

As for positively homogeneous, comonotonic additive and sub-additive risk measures nothing worse than the comonotonic case can happen, it seems natural to measure diversification by comparison with the comonotonic scenario ${ }^{10}$. This suggests the following definition.

Definition 4.1 Let $X_{1}, \ldots, X_{n}$ be real-valued random variables and let $Y=\sum_{i=1}^{n} X_{i}$. If $\rho$ is a risk measure such that $\rho(Y), \rho\left(X_{1}\right), \ldots, \rho\left(X_{n}\right)$ are defined, then

$$
\operatorname{DF}_{\rho}(Y)=\frac{\rho(Y)}{\sum_{i=1}^{n} \rho\left(X_{i}\right)}
$$

denotes the diversification factor of portfolio $Y$ with respect to the risk measure $\rho$. The fraction

$$
\operatorname{DF}_{\rho}\left(X_{i} \mid Y\right)=\frac{\rho\left(X_{i} \mid Y\right)}{\rho\left(X_{i}\right)}
$$

\footnotetext{
${ }^{10}$ Martin and Tasche (2005) suggest another approach to measuring diversification as they calculate the proportions of systematic and idiosyncratic risk within the total risk of the portfolio.
} 
with $\rho\left(X_{i} \mid Y\right)$ being the risk contribution of $X_{i}$ as in Definition 3.1 denotes the marginal diversification factor of sub-portfolio $X_{i}$ with respect to the risk measure $\rho$.

Note that without calling the concept "diversification factor", Memmel and Wehn (2006) calculate a diversification factor for the German supervisor's market price risk portfolio. Garcia Cespedes et al. (2006) $)$ use the diversification factors as defined here for a representation of portfolio risk as a "diversification factor"-weighted sum of stand-alone risks.

If $\rho$ is sub-additive and positively homogeneous, then by (4.1) both $\operatorname{DF}_{\rho}(Y)$ and $\operatorname{DF}_{\rho}\left(X_{i} \mid Y\right)$ will be bounded by 1 . If $\rho$ is additionally comonotonic additive, then the bound 1 can be reached by portfolios with comonotonic risks. Thus, with a reasonable risk measure, $\mathrm{DF}_{\rho}(Y)$ being close to 1 will indicate that there is no significant diversification in the portfolio. Similarly, a value of $\operatorname{DF}_{\rho}\left(X_{i} \mid Y\right)$ close to 1 will indicate that there is almost no diversification effect with asset $i$. As the dependence - measured as degree of comonotonicity - in a portfolio is influenced both by the idiosyncratic and the systematic risk factors, the diversification factors according to Definition 4.1 capture name diversification as well as sectoral diversification.

Although VaR is not a sub-additive risk measure in general, some authors argue that, by practical experience, it can be considered an almost sub-additive risk measure (cf. Danielsson et al., 2005). This observation, however, seems to be strongly dependent on the context. For instance, Frev and McNeil (2002, Section 2.3) present an example of a credit portfolio where "measuring risk with VaR can lead to nonsensical results". In the following section we illustrate the use of the diversification factors from Definition 4.1 by a numerical example. We consider VaR as the underlying risk measure because this facilitates the calculations.

\section{$5 \quad$ Numerical example}

In this section, we illustrate the application of the formulae for the loss distribution function (Proposition 2.5) and the risk contributions to VaR (Theorem 3.4) with a simple example. We consider a special case of model (2.10) with two normally distributed systematic factors and normally distributed idiosyncratic risk drivers as in Example 2.1

As for (2.9), we consider the case $n=2$ which may be interpreted as having a portfolio with a large number of credit instruments in two different sectors. Both of these sectors are exposed to the first systematic factor, but only the first sector is also exposed to the second factor. By varying the extent of this exposure to the second systematic factor we will obtain a picture of the effect of sectoral diversification by dependence on more than one systematic factors. Additionally, we will fix the exposure to the second systematic factor but vary the weights of the sectors within the portfolio in order to get an impression of the influence of the sectoral structure on the diversification factors defined in Section 4. 
Example 5.1 Consider the loss variable $\hat{L}(u)$ from Example 2.1 in the case $n=2$ with standard normally distributed systematic factors $S_{1}$ and $S_{2}$ and independent (also of $\left(S_{1}, S_{2}\right)$ ) standard normally distributed idiosyncratic risk drivers $\xi_{1}, \xi_{2}$. We consider the case of relative (to the total exposure) loss, i.e. the case $u_{1}+u_{2}=1$. Hence, $\hat{L}(u)$ reads here

$$
\hat{L}(u)=u \Phi\left(\frac{t_{1}-\sqrt{\varrho_{1,1}} S_{1}-\sqrt{\varrho_{1,2}} S_{2}}{\omega_{1}}\right)+(1-u) \Phi\left(\frac{\Phi^{-1}(p)-\sqrt{\varrho_{2,1}} S_{1}-\sqrt{\varrho_{2,2}} S_{2}}{\omega_{2}}\right) .
$$

With respect to the correlations with the systematic factors, we fix some $\varrho \in(0,1)$ and let

$$
\begin{array}{lll}
\varrho_{1,1}=\sqrt{\frac{\varrho w}{1+2 w(1-w) \tau}}, & \varrho_{1,2}=\sqrt{\frac{\varrho(1-w)}{1+2 w(1-w) \tau},} \\
\varrho_{2,1}=\sqrt{\varrho}, & \varrho_{2,2}=0,
\end{array}
$$

where $w \in[0,1]$ is a weight parameter controlling the exposure of the first sector to the second factor and $\tau$ denotes the correlation of $S_{1}$ and $S_{2}$, i.e. $\tau=\operatorname{corr}\left[S_{1}, S_{2}\right]$. Choosing the $\varrho_{i, j}$ as in (5.2a) and (5.2b) implies by (2.6)

$$
\omega_{1}=\sqrt{1-\varrho}=\omega_{2}
$$

The square-root representation in (5.2a) and (5.2b) was chosen in order to make the correlations comparable in size with those from BCBS (2006, $\S 272)$. By choosing $\varrho_{1,1}$ and $\varrho_{1,2}$ as in (5.2a), we ensure that in (5.1) both factor combinations $\varrho_{i, 1} S_{1}+\varrho_{i, 2} S_{2}, i=1,2$, are identically normally distributed with variance $\varrho$. As a consequence, the weights on idiosyncratic risk are the same across the two sectors. Thus, in the example all observed differences in VaR or in the risk contributions are due to the sector structure only. A weight $w=1$ means that we are in a single factor model, whereas $w=0$ implies that the factors that drive the risk of the both sectors have correlation $\tau$ and are, in particular, independent for $\tau=0$.

For the first calculations we choose

$$
t_{1}=\Phi^{-1}(0.1)=t_{2}, \quad \varrho=0.1, \quad u=0.1
$$

This choice is mainly driven by the desire to come up with illustrative results. The value $10 \%$ for $\varrho$ is somewhere in the center of the span provided by BCBS (2006). The choice for the threshold values $t_{1}$ and $t_{2}$ may be interpreted as having a large credit portfolio with two sectors, both with an average probability of default of $10 \%$.

In order to assess the impact on sectoral diversification by several systematic factors at portfolio level, first we calculate ${ }^{11}$ VaR-figures at different confidence levels both for the single factor model as in (5.1) with $w=1$ as well as for the two-factor model with $w=0$. Table 1 for independent systematic factors and Table 2 for positively correlated systematic factors show that the impact

\footnotetext{
${ }^{11}$ The calculations for the following examples require numerical root-finding and integration. Files with the R-code (cf. R Development Core Team, 2003) used by the author can be provided upon request.
} 
Table 1: VaRs at different confidence levels $\alpha$ for the asymptotic single $(w=1)$ and two-factor $(w=0)$ models as in Example [5.1. Parameter values as in (5.3). Independent systematic factors $(\tau=0)$.

\begin{tabular}{c||c|c|c|c|c|c|c}
$\alpha$ & $75 \%$ & $90 \%$ & $95 \%$ & $97.5 \%$ & $99 \%$ & $99.9 \%$ & $99.95 \%$ \\
\hline VaR (single factor) & $13.0 \%$ & $17.8 \%$ & $21.1 \%$ & $24.3 \%$ & $28.3 \%$ & $37.4 \%$ & $40.0 \%$ \\
\hline VaR (two factors) & $12.7 \%$ & $17.0 \%$ & $20.0 \%$ & $22.9 \%$ & $26.5 \%$ & $34.7 \%$ & $37.0 \%$ \\
\hline Ratio of the above & $97.9 \%$ & $95.8 \%$ & $94.9 \%$ & $94.3 \%$ & $93.7 \%$ & $92.8 \%$ & $92.6 \%$
\end{tabular}

even in the case of an independent second factor and for high levels of VaR remains limited. The results from Table 2] compared to those of Table 1] reflect the non-surprising fact that, with positively correlated systematic factors, the potential for diversification is less than in the case of independent systematic factors.

Table 2: VaRs at different levels $\alpha$ for the asymptotic single $(w=1)$ and two-factor $(w=0)$ models as in Example 5.1. Parameter values as in (5.3). Systematic factors with $50 \%$ correlation $(\tau=0.5)$.

\begin{tabular}{c||c|c|c|c|c|c|c}
$\alpha$ & $75 \%$ & $90 \%$ & $95 \%$ & $97.5 \%$ & $99 \%$ & $99.9 \%$ & $99.95 \%$ \\
\hline VaR (single factor) & $13.0 \%$ & $17.8 \%$ & $21.1 \%$ & $24.3 \%$ & $28.3 \%$ & $37.4 \%$ & $40.0 \%$ \\
\hline VaR (two factors) & $12.9 \%$ & $17.4 \%$ & $20.6 \%$ & $23.6 \%$ & $27.4 \%$ & $36.1 \%$ & $38.5 \%$ \\
\hline Ratio of the above & $99.2 \%$ & $98.1 \%$ & $97.6 \%$ & $97.3 \%$ & $96.9 \%$ & $96.4 \%$ & $96.3 \%$
\end{tabular}

The impact of sectoral diversification turns out to be much higher if we consider UL contributions with respect to VaR instead of total VaR. "UL" means "unexpected loss" and is defined by choosing

$$
\rho(V)=\operatorname{VaR}_{\alpha}(V)-\mathrm{E}[V]=q_{\alpha}(V)-\mathrm{E}[V]=\mathrm{UL}(V)
$$

in Definition 3.1. In Figure 1 we plot the relative contribution to UL with respect to 99.9\%-VaR of the first sector in the model in Example 5.1 (i.e. the ratio of the contribution of the first sector to UL in the sense of Definition 3.1 and portfolio-wide UL) against the extent of the sector's exposure to the first factor (low values of $w$ correspond to low exposure, values of $w$ close to 1 correspond to high exposure). For calculating the contributions, we applied (3.7). In the case of independent systematic factors, it turns out that the size of the risk contribution of the first sector can be reduced to almost 0 when it is exposed to the second systematic factor only. The rate of the reduction becomes the smaller the stronger the exposure to the first systematic factor but remains significant. These effects are significantly weaker, if there is a positive, but less than $100 \%$ correlation of the systematic factors. There is no reduction at all of the risk contribution, if the correlation of the systematic factors is $100 \%$ - this corresponds to the case 
of the asymptotic single risk factor model that underlies the Basel II risk capital charges.

Figure 1: Relative contribution to UL with respect to 99.9\%-Var of the first sector in the model in Example 5.1 as function of the extent of the sector's exposure (measured by $w \in[0,1])$ to the first factor. Parameter values as in (5.3). Factor correlations $50 \%, 0 \%$, and $100 \%$ respectively.

\section{1st sector contribution to total risk}

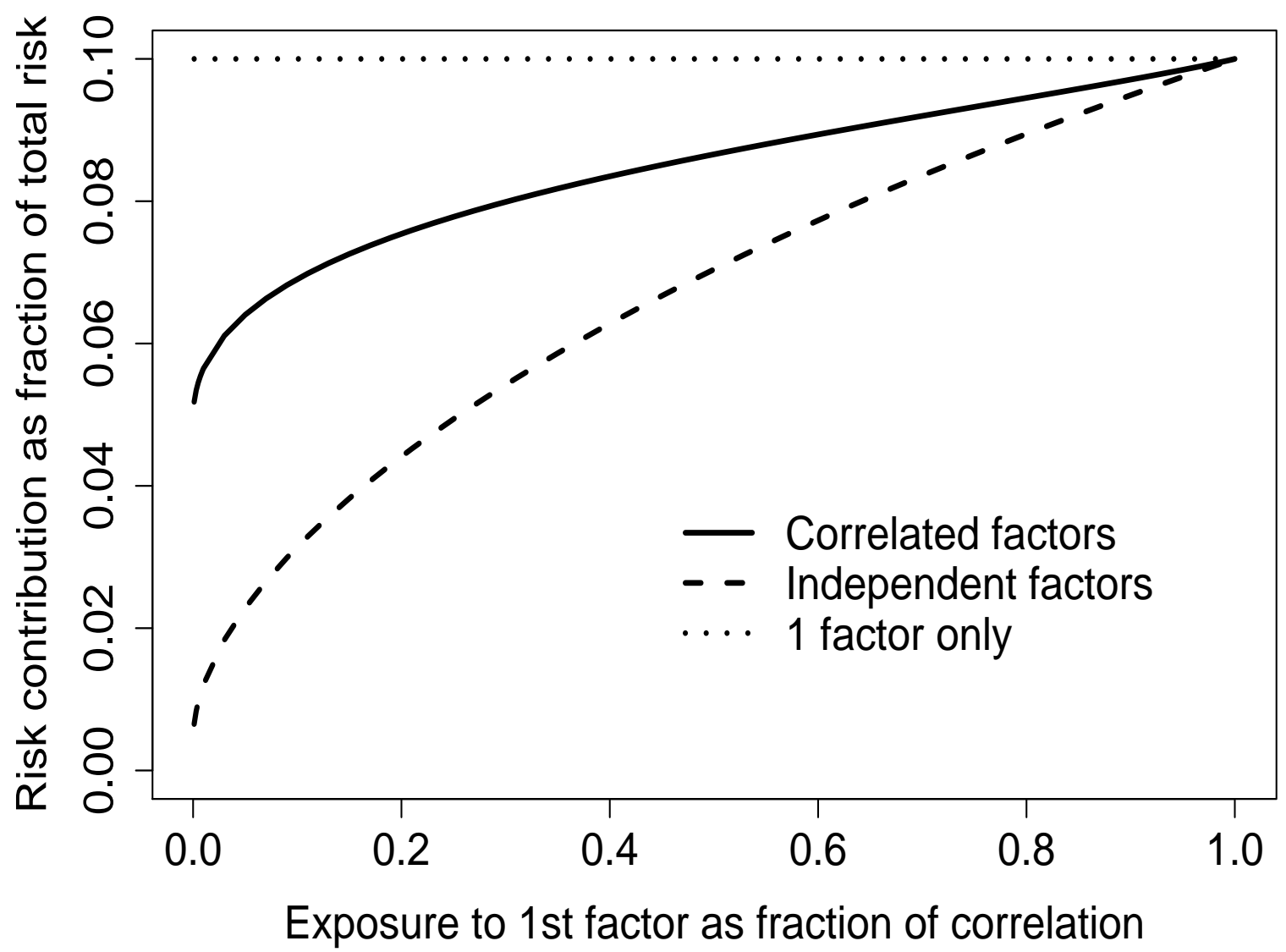

In order to illustrate the functioning of the diversification factors defined in Section 4 we fix the exposure of the first sector to the second systematic factor by setting $w=0.5$. We then make the weight $u$ (see (5.1) ) of the first sector in the portfolio move from $0 \%$ to $100 \%$. As in the case of Figure 1. we calculate with two different values for the correlation of the two systematic factors. The parameter settings for Figure 2 are given in Equations (5.5a) and (5.5b) respectively.

$$
\begin{array}{llll}
\tau=0, & t_{1}=\Phi^{-1}(0.2), & t_{2}=\Phi^{-1}(0.1), & \varrho=0.1 \\
\tau=0.5, & t_{1}=\Phi^{-1}(0.2), & t_{2}=\Phi^{-1}(0.1), & \varrho=0.1 .
\end{array}
$$

Figure 2illustrates the connection between the two types of diversification factors from Definition 4.1. In both panels, the solid line shows the portfolio-wide diversification factor of the loss 
variable from (5.1). The dashed lines in the panels of Figure 2 reflect the corresponding marginal diversification factor of the first sector, whereas the dotted lines give the marginal diversification factor of the second sector. The first panel of Figure 2 represents the case of independent systematic factors, whereas the second panel of Figure 2 shows the case of positively correlated systematic factors. From the definition of the risk contribution by means of a derivative (see Definition (3.1), in both cases it follows that the three lines intersect at just the weight of the first sector that yields the most diversified portfolio in the sense of being the portfolio with the minimum diversification factor to be feasible by changing the sector weights.

Figure 2: Diversification factors with respect to UL in sense of Definition 4.1 for loss variable $\hat{L}(u)$ and sectors from Example 5.1, with $w=0.5$. Represented as functions of weight $u \in[0,1]$ of first sector. First panel for case of independent systematic factors, second panel for case of correlated systematic factors. Parameter setting specified by (5.5a) and (5.5b) respectively.

Diversification factors: independent systematic factors

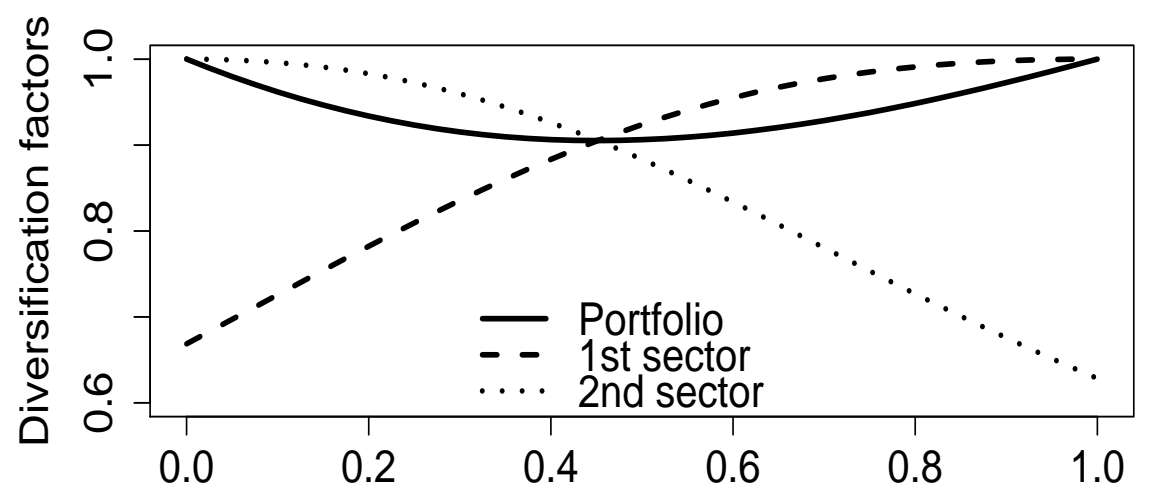

Diversification factors: correlated systematic factors

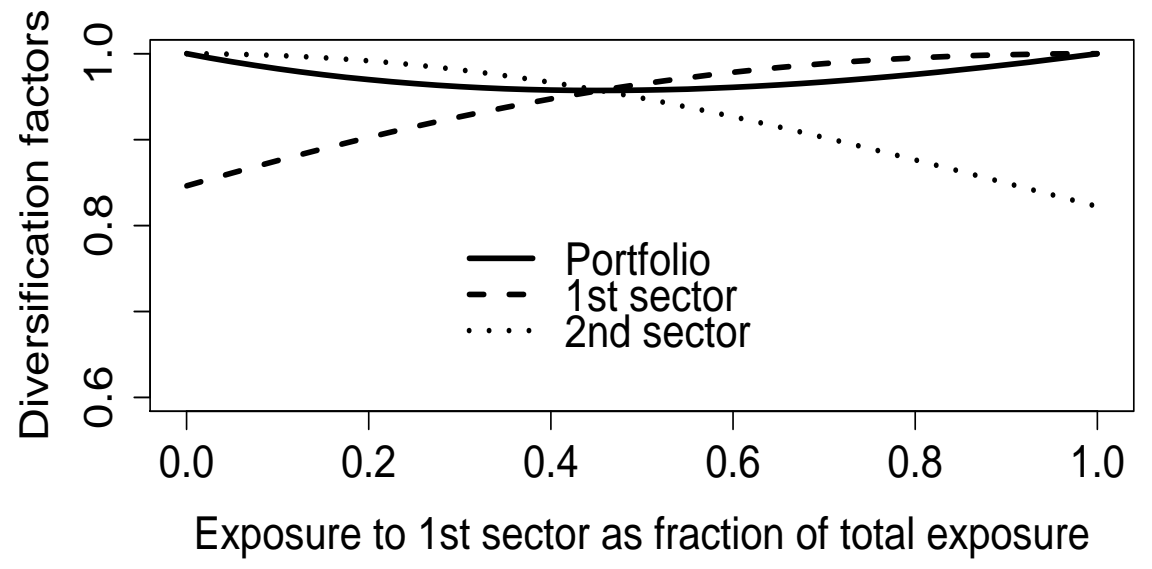

According to Figure 2, those portfolios where portfolio-wide and marginal diversification factors 
are close together could be called well-diversified. A wide range of the diversification factors of a portfolio would then indicate that the portfolio is not very well diversified. However, Figure 2 demonstrates that the possible range of the diversification factors depends on the potential for diversification. The smaller maximum possible range of the marginal diversification in the correlated case correctly indicates that in the extreme case of exposure to only one systematic factor the portfolio is closer to the optimum than it is in the independent case. But this is only due to the fact that in the case of positively correlated systematic factors, the potential for diversification is less than in the case of independent systematic factors, as follows from the higher value of the minimum portfolio-wide diversification factor.

\section{Conclusions}

In this paper we have derived closed-form formulae for risk contributions to VaR and ES in the context of asymptotic multi-factor models, thus generalizing the capital requirements as provided by the Basel II Accord in the context of the ASRF (Asymptotic Single Risk Factor) model. The effort needed for the numerical calculations is higher than in the ASRF case but, as a numerical example shows, remains feasible at least in the case of two-factor models. The example also indicates that the effect of sectoral diversification by several systematic factors on portfolio-wide economic capital is moderate but can be significant for risk contributions of single assets, sectors or sub-portfolios.

The risk contributions we have analyzed in the first sections of the paper can be used for calculating diversification factors for sub-portfolios, sectors or assets in a portfolio. If these factors, considered for all the assets, sectors of sub-portfolios in the portfolio, take a wide range, then there is a high potential for diversification in the portfolio. If, in contrast, the range of the factors is narrow, there is not much potential left for diversification by changing the weights of the assets, sectors or sub-portfolios in the portfolio. In this case, more diversification can only be reached by adding new assets or by removing assets from the portfolio.

This observation suggests the use of the newly developed diversification factors for reflecting sectoral diversification by several systematic factors: assets, sectors or sub-portfolios found welldiversified by a marginal diversification factor close to the portfolio-wide diversification factor could receive a reduction of capital requirements. The sizes of such reductions could be estimated by means of an asymptotic multi-factor model. Of course, the concrete choice of the model and its underlying parameters might have a strong impact on the estimates. Further research in this direction seems necessary. 


\section{References}

Acerbi, C. And D. TAsche (2002) On the coherence of Expected Shortfall. Journal of Banking and Finance 26(7), 1487-1503.

Artzner, P., Delbaen, F., Eber, J.-M. And D. Heath (1999) Coherent Measures of Risk. Mathematical Finance 9, 203-228.

Basel Committee on Banking Supervision (BCBS) (2006) Basel II: International Convergence of Capital Measurement and Capital Standards: a Revised Framework - Comprehensive Version. http://www. bis.org/publ/bcbs128.htm

Bluhm, C., Overbeck, L. and C. Wagner (2002) An Introduction to Credit Risk Modeling. CRC Press: Boca Raton.

Danielsson, J., Jorgensen, B. N., Sarma, M. And C. G. De Vries (2005) Sub-additivity re-examined: the case for Value-at-Risk. Working paper. http://www . eurandom.nl/reports/2005/006MSreport.pdf

Denault, M. (2001) Coherent allocation of risk capital. Journal of Risk 4(1), 1-34.

Dhaene, J., Vanduffel, S., Tang, Q., Goovaerts, M., KaAs, R. and D. Vyncke (2006) Risk measures and comonotonicity: a review. Stochastic Models 22(4), to appear.

Embrechts, P., HöIng, A. And A. Juri (2003) Using Copulae to bound the Value-at-Risk for functions of dependent risks. Finance \& Stochastics 7(2), 145-167.

Emmer, S. And D. Tasche (2005) Calculating Credit Risk Capital Charges with the OneFactor Model. Journal of Risk 7(2), 85-101.

Frey, R. AND A. MCNeIL (2002) VaR and expected shortfall in portfolios of dependent credit risks: conceptual and practical insights. Journal of Banking and Finance 26(7), 1317-1334.

Garcia Cespedes, J. C., de Juan Herrero, J. A., Kreinin, A. And D. Rosen (2006) A Simple Multi-Factor "Factor Adjustment" for the Treatment of Credit Capital Diversification. Journal of Credit Risk, this volume.

Gordy, M. (2003) A Risk-Factor Model Foundation for Ratings-Based Bank Capital Rules. Journal of Financial Intermediation 12(3), 199-232.

Gouriéroux, C., Laurent, J. P. And O. Scaillet (2000) Sensitivity analysis of Values at Risk. Journal of Empirical Finance, 7, 225-245.

Kalkbrener, M. (2005) An axiomatic approach to capital allocation. Mathematical Finance 15(3), 425-437. 
Kalkbrener, M., Lotter, H. and L. Overbeck (2004) Sensible and efficient capital allocation for credit portfolios. Risk 17(1), S19-S24.

Lemus, G. (1999) Portfolio Optimization with Quantile-based Risk Measures. PhD thesis, Sloan School of Management, MIT. http://citeseer.ist.psu.edu/lemus99portfolio.html

Litterman, R. (1996) Hot Spots ${ }^{\mathrm{TM}}$ and Hedges. The Journal of Portfolio Management 22, $52-75$.

Lucas, A., Klaassen, P., Spreiu, P. and S. Straetmans (2001) An analytic approach to credit risk of large corporate bond and loan portfolios. Journal of Banking $\mathscr{G}$ Finance 25, $1635-1664$.

Luciano, E. AND M. MAREnA (2003) Value at risk bounds for portfolios of non-normal returns. In New Trends in Banking Management. C. Zopoudinis (ed.). Physica-Verlag, 207222 .

Martin, R. And D. Tasche (2005) CVaR: A Tale of Two Parts. Working paper.

Mausser, H. And D. Rosen (2004) Allocating Credit Capital with VaR Contributions. Working paper, Algorithmics Inc.

Memmel, C. And C. Wehn (2006) The supervisor's portfolio: the market price risk of German banks from 2001 to 2004 - Analysis and models for risk aggregation. Journal of Banking Regulation 7, 309-324.

Myers, S. C. And J. A. Read (2001) Capital Allocation for Insurance Companies. The Journal of Risk and Insurance 68, 545-580.

Patrik, G., Bernegger, S. And M.B. Rüegg (1999) The use of risk adjusted capital to support business decision-making. CAS Forum 1999 Spring, Reinsurance Call Papers. http://www. casact.org/pubs/forum/99spforum/99spftoc.htm

Pykhtin, M. (2004) Multi-factor adjustment. Risk 17(3), 85-90.

R Development Core Team (2003) R: A language and environment for statistical computing. R Foundation for Statistical Computing, Vienna. http://www.R-project.org

Tasche, D. (1999) Risk contributions and Performance Measurement. Working paper, Technische Universität München.

TAsche, D. (2002) Expected Shortfall and Beyond. Journal of Banking and Finance 26(7), 1519-1533. 